\title{
Revisión del tratamiento dietético-nutricional de la anorexia nerviosa
}

'Área de Nutrición y Bromatología. Universidad Pablo de Olavide. Sevilla. España. ${ }^{2}$ Instituto de Ciencias de la Conducta. Sevilla. España.

Financiación: ninguna.

Recibido el 22 de noviembre de 2010, aceptado el 13 de julio de 2011.

Correspondencia a: Ignacio Jáuregui-Lobera Virgen del Monte 31

41011-Sevilla (España)

Teléfono: +34954280781

Fax: +34954278167

E-mail: ijl@tcasevilla.com

\author{
IGNACIO JÁUREGUI-LOBERA, PATRICIA BOLAÑOS-RÍOS²
}

\section{Review of nutritional and dietary management of anorexia nervosa}

While numerous studies have highlighted the need to approach anorexia nervosa from a multidisciplinary perspective, the dietary and nutritional aspects of the disorder are rarely considered in depth. Basic guidelines to monitor food intake of patients, are available. A literature review was performed in Medline, searching for articles related to the dietary and nutritional management of anorexia nervosa and published over the last five years. The final analysis focused on 102 articles. This review summarizes the different therapeutic contexts and objectives, nutritional support, the use of dietary supplements, the role of artificial nutrition, refeeding syndrome, involuntary treatment and nutritional education.

(Rev Med Chile 2012; 140: 98-107).

Key words: Diet therapy; Dietary supplements; Refeeding syndrome.
L a anorexia nerviosa es un trastorno habitualmente de curso prolongado, con manifestaciones psicofísicas diversas ${ }^{1}$. Un porcentaje de pacientes puede morir, por suicidio o complicaciones físicas, siendo frecuente la muerte súbita por cardioarritmias ${ }^{1-3}$, que no guarda relación con la duración de la enfermedad o la edad, pero sí con un índice de masa corporal (IMC) $<12$ y niveles muy bajos de albúmina sérica ${ }^{4}$, aunque el riesgo de dicho desenlace es poco predecible, dada la variable respuesta individual a la desnutrición y la influencia de otros factores ${ }^{5,6}$. En las complicaciones influyen el bajo peso, atracones, conductas purgativas, ejercicio extenuante y autolesiones, siendo reversibles con una adecuada recuperación ponderal ${ }^{7}$. La prevalencia del trastorno es $0,2-0,5 \%{ }^{8}$, afectando más a las mujeres ${ }^{9}$, pero con iguales efectos en los varones ${ }^{10}$. Aún sin demostrar que la ratio mujeres-hombres esté cambiando, lo que sí ha aumentado es el rango de edad de aparición, considerando que el rango tradicional de afectación era de 15 a 24 años para la mayoría de los casos ${ }^{1}$.
La morbi-mortalidad asociada y la compleja naturaleza del trastorno, implican un tratamiento interdisciplinar ${ }^{11}$, muchas veces intensivo ${ }^{12}$, siendo los dietistas-nutricionistas los mejor cualificados para manejar aspectos dietético-nutricionales ${ }^{13}$, como el restablecimiento ponderal, el logro de una ingesta que cubra los requerimientos y una adecuada educación nutricional ${ }^{4}$.

El objetivo del trabajo fue revisar los aspectos dietético-nutricionales del tratamiento de la anorexia nerviosa, enfatizando las tareas y estándares necesarios a proporcionar por parte de los dietistas-nutricionistas en el contexto de un equipo interdisciplinar $\mathrm{y}$ en diferentes ámbitos: ambulatorio, hospitalario y de hospitalización parcial (unidades de día).

\section{Material y Métodos}

Se hizo una revisión selectiva de las publicaciones indizadas en PubMed, sin carácter de revisión sistemática ni metaanálisis. Se incluyeron trabajos relacionados con el tratamiento dietético- 
Tratamiento nutricional de la anorexia nerviosa - I. Jáuregui-Lobera et al

nutricional, publicados en los últimos cinco años, en la base de datos Medline, introduciendo los siguientes términos: tratamiento dietético de la anorexia nerviosa, dietética y anorexia nerviosa, nutrición y anorexia nerviosa, educación nutricional y anorexia nerviosa, tratamiento nutricional y anorexia nerviosa.

\section{Resultados}

Se encontraron 412 artículos, eliminándose 310 , tras la lectura de todos los resúmenes, al no ser trabajos relacionados con el propósito del estudio, analizando 102 artículos, incluyendo 15 artículos y textos relevantes, independientemente de su fecha de publicación.

\section{El tratamiento actual de la anorexia nerviosa}

El tratamiento comporta la rehabilitación y consejo nutricionales, psicoterapia individual e intervenciones familiares, fundamentalmente psicoeducativas $^{14}$. Además, desde el primer momento los médicos de atención primaria juegan un importante papel desde el punto de vista dietéticonutricional ${ }^{15}$. El tratamiento médico, psicológico y nutricional se contempla siempre, independientemente de la severidad del cuadro ${ }^{7}$, siendo lo más controvertido el uso de medicación, por cuanto se han utilizado innumerables fármacos con escasos resultados ${ }^{16}$. En ocasiones, se ha observado alguna mejoría (en la conducta alimentaria, estado de ánimo y síntomas obsesivos), especialmente si hay comorbilidad psiquiátrica asociada y en niños y adolescentes ${ }^{17}$. En todo caso, los problemas metodológicos, asociados a la mayoría de estudios, impiden hacer afirmaciones claras sobre su utilidad ${ }^{18}$. Los aspectos dietético-nutricionales del tratamiento incluyen recuperación ponderal, plan dietético personalizado, corrección de la baja ingesta de alimentos y corrección de la percepción de los pacientes sobre lo que es una dieta saludable, siendo relevantes para ello el diagnóstico precoz y la intervención nutricional desde el comienzo ${ }^{19}$. Controvertida también es la participación de los pacientes en la elección de su dieta, pues la necesidad de recuperación ponderal y de normalización de hábitos obliga a ciertas imposiciones, en principio, innegociables, ya que cuando los pacientes eligen la dieta, al menos durante cierto tiempo, se acercan más a patrones inadecuados, habituales en la población, que a los objetivos terapéuticos ${ }^{20}$. La Figura 1 muestra los principales componentes de la intervención dietético-nutricional en la anorexia nerviosa.

\section{El contexto terapéutico}

El tratamiento ambulatorio exige situación médica estable, ausencia de riesgo suicida y adecuada capacidad para la vida familiar, académica o laboral. La mayoría de pacientes con trastornos alimentarios que requieren ingreso padecen anorexia nerviosa, siendo indicaciones para ello las de naturaleza médica (entre ellas un peso inferior al

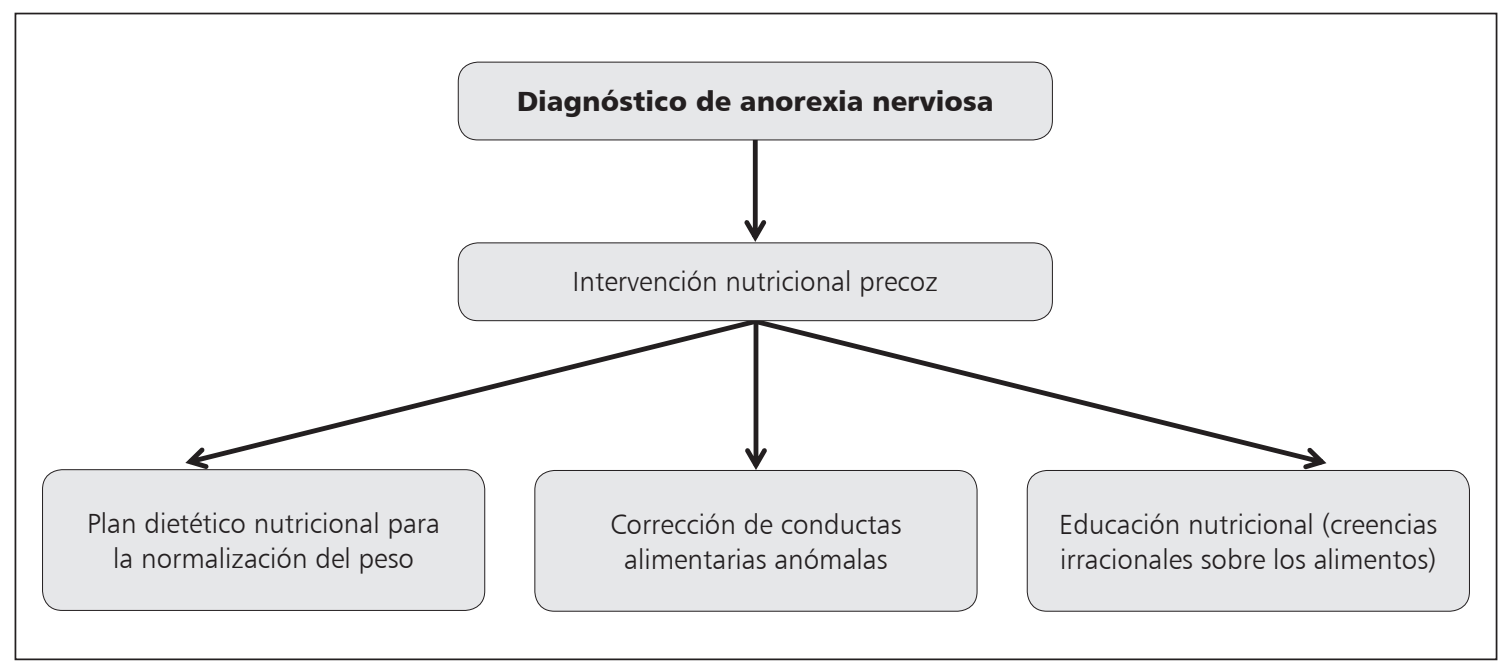

Figura 1. Principales componentes de la intervención dietético-nutricional en la anorexia nerviosa. 
$75 \%$ del estimado normal o una aguda pérdida de peso con rechazo a comer) o las que tienen que ver con patología psiquiátrica asociada ${ }^{21,22}$. En relación con el IMC, se ha propuesto que para un tratamiento ambulatorio dicho IMC debería ser $\geq$ 16 y para un ingreso hospitalario (a veces urgente) $\leq 13,9$. La decisión en otros casos recaería en otros criterios $^{4}$, no siendo el riesgo físico, medido a través del IMC, el único elemento de decisión ${ }^{5}$. Los programas de hospitalización parcial (unidades de día) son efectivos en cuanto a la ganancia de peso y la mejoría de algunos síntomas, pero hay pocos estudios controlados al respecto, así como sobre los criterios de alta de dichos programas ${ }^{21,23-25}$.

\section{Objetivos terapéuticos}

La recuperación ponderal es un objetivo primordial ${ }^{26}$, aconsejándose el ingreso cuando el peso es inferior al $75 \%$ del ideal ${ }^{27}$. Los requerimientos energéticos variarán dependiendo, por ejemplo, del IMC y de la masa grasa corporal inicial ${ }^{26}$, de manera que si el IMC es $<14$ y la masa grasa $<4$ kilos, la recuperación es mayor mediante el tratamiento dietético. En todo caso, dado que influyen muchos factores (reposición de fluidos, adecuado aporte energético, etc.) la mayor o menor ganancia ponderal es bastante impredecible ${ }^{4}$, existiendo, además, una falta de consenso al fijar el objetivo de recuperación en adolescentes en desarrollo, habiéndose propuesto el uso de percentiles de IMC y fijado un rango entre 14 y 39 como objetivo inicial $^{28}$. La inadecuada recuperación ponderal, actitudes alimentarias anómalas y baja edad, se han relacionado con rehospitalizaciones ${ }^{29}$, siendo la adecuada ganancia ponderal el mejor predictor, a corto plazo, de menor deterioro clínico ${ }^{30}$. Un mejor peso inicial también se ha relacionado positivamente con un mejor mantenimiento posterior del mismo ${ }^{31}$ y, a largo plazo, la normalización ponderal y del estado nutricional parecen también determinantes de un buen pronóstico ${ }^{32}$.

Suelen considerarse una alimentación inicial (una a dos semanas), para mejorar el estado general del paciente y la tolerancia a la ingesta, y una alimentación posterior dirigida a la recuperación ponderal ${ }^{4}$. Aunque no hay consenso, suele comenzarse con bajos aportes energéticos en situación de ingreso (por ejemplo entre 500-700 Kcal./día), a la vez que se tiene en cuenta una adecuada reposición hidroelectrolítica ${ }^{22,33}$. De hecho el paradigma actual no persigue la rapidez en la recuperación ponderal ${ }^{34}$, que puede provocar severas complicaciones $^{35}$, sino una realimentación gradual, con más o menos diferencias individuales, en la que es habitual que no haya sustancial ganancia de peso hasta pasadas 2-3 semanas, tras lo cual acontece una estabilización y reducción de riesgos para el paciente, que dará paso a un incremento en el aporte calórico semanal hasta llegar a 2.500-3.000 Kcal., aspirando a una ganancia semanal, que se estima ideal (y recomendada) en unos $500 \mathrm{~g}^{22}$. El aporte inicial suele ser de 30-40 Kcal/Kgr/día para ir aumentando hasta lograr esa ganancia semanal ideal ${ }^{36}$. Hay consenso en el hecho de que las recuperaciones ponderales muy rápidas $(>700$ $\mathrm{g} /$ semana) asocian prematuras pérdidas de peso tras el alta ${ }^{37}$, lo que justifica la gradualidad antes señalada ${ }^{34}$. Figura 2.

El uso de suplementos hipercalóricos ha mostrado alguna utilidad, especialmente en la primera parte del proceso de realimentación ${ }^{38}$, mientras que otros suplementos de vitaminas y minerales no resultan útiles para la recuperación ponderal ${ }^{39}$.

\section{El soporte nutricional en la anorexia nerviosa}

El manejo de la realimentación y de las complicaciones que pueden derivarse (por ejemplo, el síndrome de realimentación) parece bien consensuado $^{33,40-43}$.

En principio, la recuperación del peso normal debe lograrse mediante una alimentación normal y adecuado soporte nutricional ${ }^{4}$. Éste, dados los problemas psicológicos asociados y la frecuente presencia de dispepsia funcional, debe incluir apoyo psicológico ${ }^{44-48}$. Además de la recuperación ponderal, los pacientes deben recuperar un patrón normal de alimentación. El contexto terapéutico (ambulatorio, hospitalización o unidades de día) marca algunas variaciones en cuanto al ritmo y forma de lograr los objetivos señalados ${ }^{36}$.

El plan inicial contempla la introducción de alimentos de los principales grupos y que los pacientes comiencen a comer, pues no es posible, por la resistencia de los mismos, pretender cubrir, desde el principio, todos los requerimientos (en relación con la situación de desnutrición, pero también en función de la edad, sexo, etc. ${ }^{4,36}$. La ganancia de peso, siguiendo pasos graduales mediante una alimentación normal, se considera el elemento más importante en el tratamiento de la anorexia nerviosa ${ }^{49}$.

El apoyo durante la alimentación normal 


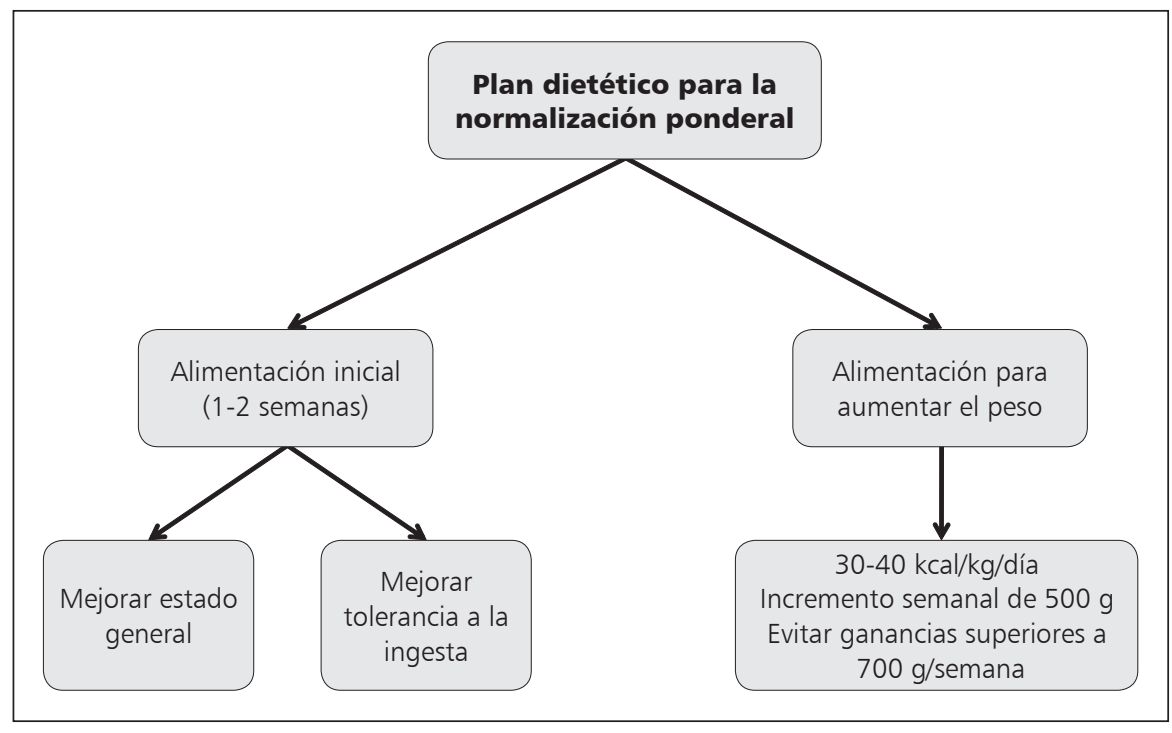

Figura 2. Pautas para la recuperación ponderal. contempla que los grupos de alimentos no son negociables, la presencia de algún miembro del equipo durante la ingesta y un tiempo concreto para la realización de las comidas. El miembro del equipo que supervisa la comida anima a su correcta realización en ese tiempo y procura introducir elementos de distracción, evitando hablar de calorías, dietas, comentarios sobre la comida, etc. La supervisión tiende a prolongarse un tiempo (por ejemplo, una hora) una vez finalizada la ingesta ${ }^{4,22,50,51}$.

Pueden negociarse alimentos de cada grupo, de manera que, por ejemplo, siempre que se tome una cantidad adecuada de proteínas animales, el paciente puede elegir hacerlo a base de carne, pescado o huevos, con la supervisión del dietista, quien establecerá los criterios de normalidad en cuanto a alimentos y porciones o raciones de los mismos, a la par que abordará los pensamientos irracionales de los pacientes acerca de la alimentación, planificación de comidas, compra de alimentos y tipos de técnicas culinarias ${ }^{52,53}$. En todo caso, la elección siempre será supervisada por el dietista para que el paciente no se acerque a patrones inadecuados en el proceso de recuperación ${ }^{20}$.

\section{Uso de suplementos}

En general los suplementos no aportan grandes beneficios, pudiendo reforzar las conductas de evitación frente a alimentos normales ${ }^{4}$. No obstante, dado que en la anorexia nerviosa se han descrito innumerables carencias nutricionales, en dicha situación podrían necesitarse. Así ocurre con el calcio ante el rechazo de los lácteos ${ }^{36}$, el hierro en pacientes vegetarianos y/o con excesivo ejercicio $^{54,55}$, el zinc si hay escasa ingesta de carne y/o ejercicio excesivo ${ }^{56,57}$, la tiamina y el ácido fólico si existe abuso de alcohol ${ }^{58,59}$ y la vitamina D si hay rechazo de lácteos y/o baja exposición solar ${ }^{60}$. En relación con los déficits de tiamina se han recogido complicaciones como el síndrome de Wernicke ${ }^{61}$. Con respecto a otros micronutrientes hay resultados dispares, que no permiten establecer pautas generales $^{62,63}$.

Uno de los tempranos indicadores de deficiencia nutricional en la anorexia nerviosa es la baja concentración plasmática de aminoácidos, como L-arginina, con resultados positivos (relacionados con la producción plaquetaria de óxido nítrico) mediante la suplementación al respecto ${ }^{64}$. En ningún caso, el uso de suplementos puede sustituir a una alimentación normal y tampoco ha mostrado eficacia para mejorar el efecto de la medicación ${ }^{39}$. La suplementación debería considerarse en pacientes embarazadas, tras la retirada de nutrición artificial y como complemento de la alimentación en pacientes con excesiva saciedad ${ }^{4,65}$. Finalmente, en relación con el sistema inmunitario, la inclusión de yogur en la alimentación mejora algunos marcadores de dicho sistema ${ }^{66}$. En la Tabla 1 se muestra un resumen de algunas de las principales indicaciones de la suplementación dietética. 
Tabla 1. Posibles indicaciones para la suplementación dietética

\begin{tabular}{|l|}
\hline $\begin{array}{l}\text { Suplementos hipercalóricos para recuperación de peso al } \\
\text { inicio del tratamiento }\end{array}$ \\
\hline Calcio si hay rechazo a la toma de productos lácteos \\
\hline $\begin{array}{l}\text { Hierro en pacientes vegetarianos y/o con excesivo ejercicio } \\
\text { físico }\end{array}$ \\
\hline $\begin{array}{l}\text { Zinc en pacientes con escasa ingesta de carne y/o con } \\
\text { excesivo ejercicio físico }\end{array}$ \\
\hline $\begin{array}{l}\text { Tiamina y ácido fólico si se asocian problemas relaciona- } \\
\text { dos con abuso de alcohol }\end{array}$ \\
\hline $\begin{array}{l}\text { Vitamina D si hay rechazo de productos lácteos y/o baja } \\
\text { exposición solar }\end{array}$ \\
\hline Arginina según concentración plasmática \\
\hline Especial atención en pacientes anoréxicas embarazadas \\
\hline $\begin{array}{l}\text { Tras alimentación artificial y en pacientes con excesiva } \\
\text { sensación de saciedad ante la alimentación normal }\end{array}$ \\
\hline
\end{tabular}

\section{Nutrición artificial}

La decisión de utilizar nutrición enteral es difícil, comportando un alejamiento psicosocial de la alimentación normal ${ }^{4,67}$. Dada la habitual ambivalencia de los pacientes ante el tratamiento, la alimentación enteral debería ser un último recurso, pues la alimentación normal proporciona habilidades, conductas y estrategias psicosociales que no se promueven con la alimentación enteral ${ }^{44}$.

Sobre su eficacia en anorexia nerviosa, hay pocos estudios, muy dispares metodológicamen$\mathrm{te}^{68-74} \mathrm{y}$ ninguno de ellos a largo plazo. Un reciente estudio, de un año de seguimiento tras nutrición enteral cíclica durante dos meses $^{75}$, concluye que la nutrición enteral es útil para la recuperación ponderal, no interfiere con la terapia de conducta alimentaria y no provoca recaídas más precoces. La aceptación de esta modalidad de tratamiento, reduce la estancia hospitalaria y no afecta a la relación terapeuta-paciente ni al grado de satisfacción de los pacientes ${ }^{76}$.

Sus indicaciones son un bajo peso con riesgo vital (IMC < 12), riesgo físico elevado y pobre aceptación de una alimentación normal a partir de una recuperación ponderal insuficiente ${ }^{77}$. Un retraso del vaciado gástrico o algunas alteraciones de motilidad intestinal, que dificultan una alimentación oral normal, pueden ser indicaciones de nutrición enteral. Los estudios realizados difieren en cuanto al hincapié que hacen en aspectos psicológicos y nutricionales del tratamiento, siendo necesario que las indicaciones de nutrición artificial los contemplen, incluyendo la definición de riesgo vital a la hora de aplicar esta modalidad de tratamiento, grado de aceptación del tratamiento, tipo de tratamiento (enteral o parenteral), indicadores de eficacia o resultados esperados durante el seguimiento y efectos (psicológicos, en función de la edad, etc. $)^{71}$.

La nutrición parenteral plantea más controversia, que se plantea cuando la gravedad del paciente lo requiere (riesgo vital) y otros medios de realimentación (oral, enteral) han fracasado ${ }^{78}$. No obstante, aunque se defiende su uso en ciertas circunstancias, como determinadas complicaciones clínicas, falta de aceptación de otras alternativas de realimentación o contraindicaciones de nutrición enteral ${ }^{79}$, se ha criticado su uso en la anorexia nerviosa, incluso su adecuación ética ${ }^{80}$. En cualquier caso, como en otros problemas médicos, la nutrición parenteral debe usarse cuando no se pueda, o no se deba, utilizar el tubo digestivo como vehículo de alimentación ${ }^{33,78,81,82}$.

\section{El síndrome de realimentación}

El síndrome de realimentación se considera una de las más graves complicaciones de la anorexia nerviosa ${ }^{35}$, incluso con resultado de muerte, tanto durante la alimentación oral, como enteral y parenteral ${ }^{83}$, debido a una excesivamente rápida o desequilibrada realimentación de pacientes severamente desnutridos ${ }^{84,85}$, y entre cuyos principales signos y síntomas se encuentran la retención de sodio y agua (con edema y posible fallo cardiaco), hipofosfatemia por alteración de procesos celulares (con afectación generalizada de sistemas y dificultad para almacenar energía), depleción de electrolitos, como potasio y magnesio, y vitaminas (con las consiguientes manifestaciones clínicas) y depleción del cofactor de tiamina, capaz de provocar un síndrome de Wernicke y/o cardiomiopatía ${ }^{7,86}$. El aporte calórico con el que puede llegar a producirse varía, en los casos comunicados, entre 10 y $60 \mathrm{Kcal} / \mathrm{Kgr} /$ día (O’Connor, 2010) ${ }^{87}$ y la presencia de glucosa en el tubo digestivo en las situaciones de extrema desnutrición parece determinante de su desencadenamiento ${ }^{87,88}$.

Para su prevención se contemplan muchas pautas de actuación médica y de enfermería ${ }^{33,40-42,89,90}$, siendo las más relevantes: a) procurar 
Tratamiento nutricional de la anorexia nerviosa - I. Jáuregui-Lobera et al

una ganancia ponderal de aproximadamente 500 grs/semana ${ }^{47}$; b) monitorizar de forma permanente el ingreso de fluidos; c) administrar fósforo y potasio durante las primeras semanas; d) valorar el posible uso de suplementos hipercalóricos y e) adecuar el aporte calórico al gasto metabólico basal inicial ${ }^{7}$, así como el aporte de hidratos de carbono $^{87,91}$.

\section{Tratamiento forzado de la anorexia nerviosa}

La expresión tratamiento forzado suele referirse al ingreso involuntario y puede confundirse con alimentación forzada, aunque el primero no debe implicar lo segundo, siendo preferible, tras un ingreso involuntario, el trabajo del personal de enfermería en lo que a la realimentación se refiere ${ }^{92}$. De hecho, se ha señalado que la alimentación enteral forzada puede dañar la relación terapéutica ${ }^{93}$, si bien hay también opiniones que han defendido lo contrario en casos de extrema necesidad ${ }^{94}$. La alimentación forzada parece contemplarse como un último recurso dado el conflicto que supone entre la autonomía de los pacientes y la obligación asistencial del equipo terapéutico, involucrando además, en muchos casos, a la familia de aqué$\operatorname{llos}^{44,95}$. En muchas ocasiones, el rechazo de un paciente a ser alimentado no es un problema de falta de capacidad, sino de falta de competencia (el paciente conoce los riesgos de no ser alimentado y tiene capacidad para razonar, pero rechaza el tratamiento, por miedos de muy diversa índole), por lo que a la hora de valorar la posible alimentación forzada debe tenerse en cuenta no sólo la capacidad sino, ante todo, la competencia, algo, por otra parte, no bien delimitado ${ }^{44,96}$.

En general, el tratamiento forzado es más probable en pacientes crónicos, con ingresos repetidos, con patología psiquiátrica asociada, bajo IMC y más patología médica asociada (lo que conlleva más probabilidad de padecer un síndrome de realimentación). En estos pacientes resulta mucho más probable la alimentación forzada ${ }^{97}$, aunque en general este tipo de tratamiento forzado suele usarse moderadamente en las unidades de tratamiento ${ }^{98}$.

\section{Educación nutricional}

Suele asumirse que la intervención psicológica puede corregir los hábitos alimentarios, pero hay claras evidencias de que eso no es posible sin una adecuada intervención nutricional, que requiere la participación de profesionales con adecuada

\section{Tabla 2. Elementos básicos del tratamiento dietético-nutricional de la anorexia nerviosa}

El tratamiento dietético-nutricional debe contemplarse en todos los casos de anorexia nerviosa, sea cual sea el contexto terapéutico, incluso desde atención primaria $7,14,15,19,36$

La mejoría en la conducta alimentaria inducida por el uso de psicofármacos no está bien establecida ${ }^{16-18}$

Determinados aspectos del tratamiento dietético-nutricional no son negociables con los pacientes 4,22,50,51,20

Aunque la decisión sobre el contexto terapéutico (ambulatorio, hospitalización, unidades de día) depende de diversos factores, el estado nutricional de los pacientes es determinante para la toma de dicha decisión $n^{4,5,21-23,25}$

La recuperación ponderal es un objetivo primordial del tratamiento dietético-nutricional4,26-28

La falta de una adecuada recuperación ponderal guarda relación con un peor curso de la enfermedad $29-32$

El abordaje dietético-nutricional debe hacerse de forma progresiva, sin ritmos acelerados 4,22,23,34-37,49

La monitorización de las comidas es esencial en la intervención dietético-nutricional4,22,33,40-48,50,51

El uso de suplementos parece tener una eficacia limitada y sólo en determinados $\operatorname{casos}^{4,36,38,39,54-65}$

El uso de nutrición artificial debe quedar sometido a una adecuada deliberación y sólo en casos de extrema necesidad ${ }^{4,44,67-83}$

El síndrome de realimentación es una de las graves complicaciones durante el tratamiento, sea cual sea la modalidad de alimentación, y, fundamentalmente, exige medidas de prevención $n^{7,33,35,40-42,47,84-92}$

El tratamiento forzado conlleva, en la mayoría de los casos, la alimentación forzada y es más frecuente en casos crónicos, con ingresos repetidos, patología psiquiátrica y médica asociadas y bajo índice de masa corporal. En todo caso debe considerarse un último recurso y someterse a una adecuada deliberación $n^{44,93-99}$

Un adecuado programa de educación nutricional es imprescindible, pues la sola intervención psicológica no logra corregir las conductas alimentarias alteradas en los pacientes ${ }^{100-102}$ 
formación al respecto. De hecho, la modificación del comportamiento alimentario puede lograrse mediante un adecuado programa de educación nutricional ${ }^{99-101}$, con lo que se han logrado mejorías en distintos aspectos relacionados con los hábitos alimentarios de los pacientes: a) mejor estructura de las comidas; b) aumento significativo del consumo de lácteos, verduras, cereales, aceite, carnes $\mathrm{y}$ frutas; c) incremento significativo en la ingesta energética y en el consumo de hidratos de carbono; d) aumento significativo en la ingesta de vitamina $\mathrm{B}_{2}$, ácido fólico, calcio y magnesio, y d) importante mejoría del estado nutricional ${ }^{102}$.

\section{Conclusiones}

La intervención nutricional en la anorexia nerviosa es esencial para su buen curso. Con variaciones en función del estado de los pacientes, el tratamiento dietético-nutricional es aplicable, desde atención primaria, en todos los contextos terapéuticos, teniendo como objetivos principales la normalización del peso y la implantación de unos adecuados hábitos de alimentación. Para ello es preferible la alimentación oral, progresiva y monitorizada, sin que la suplementación y la nutrición artificial sean pautas habituales de actuación, quedando reservadas para indicaciones concretas. Durante la intervención nutricional, el síndrome de realimentación debe tenerse en cuenta, por su especial gravedad, para adoptar las medidas preventivas pertinentes. En cuanto a la alimentación forzada, sólo debería indicarse en casos de especial gravedad, por su evolución, estado nutricional y complicaciones psiquiátricas y/o médicas. Los elementos esenciales de la intervención dietético-nutricional en la anorexia nerviosa pueden verse en la Tabla 2.

\section{Referencias}

1. Birmingham CL, Beumont P. Medical management of eating disorders. Cambridge: Cambridge University Press; 2004.

2. Strober M, Freeman R, Morrell W. The long-term course of severe anorexia nervosa in adolescents: Survival analysis of recovery, relapse, and outcome predictors over 10-15 years in a prospective study. Int J Eat Disord 1997; 22: 339-60
3. Steinhausen HC. The outcome of anorexia nervosa in the 20th century. Am J Psychiatry 2002; 159: 1284-93.

4. Cockfield A, Philpot U. Feeding size 0 : the challenges of anorexia nervosa. Managing anorexia from a dietitian's perspective. Proc Nutr Soc 2009; 68: 281-8.

5. NHS Quality Improvement Scotland. Eating Disorders in Scotland: Recommendations for management and treatment. Edimburgh: NHS QIS; 2007.

6. Keys A, Brozek J, Henschel A, Mickelsen O, Taylor HL. The biology of human starvation. Minneapolis: University of Minnesota Press; 1950.

7. Gentile MG, Pastorelli P, Ciceri R, Manna GM, Collimedaglia S. Specialized refeeding treatment for anorexia nervosa patients suffering from extreme undernutrition. Clin Nutr. 2010; 21: doi:10.1016/j.clnu.2010.03.008.

8. American Psychiatric Association. Diagnostic and statistical manual of mental disorders. 4th ed., text rev.). Washington DC: APA; 2000.

9. Thompson JK, Kinder B. Eating disorders. In: Hersen M, Turner S, editors. Handbook of adult psychopathology. New York: Plenum Press; 2003. p. 555-82.

10. Olivardia R, Harrison GP, Mangweth B, Hudson JI. Eating disorders in college men. Am J Psychiatry 1995; 152: 1279-85.

11. Mehler PS, Anderson AE. Eating disorders: A guide to medical care and complications. Baltimore: The John Hopkins University Press; 1999.

12. Guarda AS, Heinberg LJ. Inpatient and partial hospital approaches to the treatment of eating disorders. In: Thompson K, editor. Eating disorders and obesity. Hoboken, New Jersey: John Wiley \& Sons; 2004. p. 297-320.

13. Blank S, Zadik Z, Katz I, Mahazri Y, Toker I, Barak I. The emergence and treatment of anorexia and bulimia nervosa. A comprehensive and practical model. Int J Adolesc Med Health 2002; 14: 257-60.

14. Herpertz-Dahlmann B, Salbach-Andrae H. Overview of treatment modalities in adolescent anorexia nervosa. Child Adolesc Psychiatr Clin N Am 2009; 18: 131-45.

15. Miján de la Torre A, Pérez-García A, Martín de la Torre E, de Mateo Silleras B. Is an integral nutritional approach to eating disorders feasible in primary care? $\mathrm{Br} \mathrm{J}$ Nutr 2006; 96 Suppl 1: S82-5.

16. Crow SJ, Mitchell JE, Roerig JD, Steffen K. What potential role is there for medication treatment in anorexia nervosa? Int J Eat Disord 2009; 42: 1-8.

17. Rossi G, Balottin U, Rossi M, Chiappedi M, Fazzi E, Lanzi G. Pharmacological treatment of anorexia nervosa: a retrospective study in preadolescents and adolescents. Clin Pediatr (Phila) 2007; 46: 806-11.

18. Attia E, Schroeder L. Pharmacologic treatment of anorexia nervosa: Where do we go from here? Int J Eat 
Disord 2005; 37: S60-S63.

19. Lim S L, Sinaram S, Ung E K, Kua E H. The pursuit of thinness: an outcome study of anorexia nervosa. Singapore Med J 2007; 48: 222-6.

20. Jáuregui Lobera I, Bolaños Ríos P. Choice of diet in patients with anorexia nervosa. Nutr Hosp 2009; 24: 682-7.

21. Halmi KA. Salient components of a comprehensive service for eating disorders. World Psychiatry 2009; 8: 150-5.

22. National Institute for Health and Clinical Excellence. Eating disorders: Core interventions in the treatment and management of anorexia nervosa, bulimia nervosa and related eating disorders. London: National Institute for Clinical Excellence; 2004.

23. Dancyger I, Fornari V, Schneider M. Adolescents and eating disorders: an example of a day treatment program. Eat Weight Disord 2003; 8: 242-8.

24. Heinberg A, Haug N, Freeman Y. Clinical course and short-term outcome of hospitalized adolescents with eating disorders: The success of combining adolescents and adults on an eating disorder unit. Eat Weight Disord 2003; 8: 326-31.

25. Zipfel S, Reas D, Thornton C. Day hospitalization programs for eating disorders: A systematic review of the literature. Int J Eat Disord 2002; 31: 105-17.

26. Yamashita S, Kawai K, Yamanaka T, Inoo T, Yokoyama $\mathrm{H}$, Morita Ch, et al. BMI, body composition, and the energy requirement for body weight gain in patients with anorexia nervosa. Int J Eat Disord 2010; 43: 365-71.

27. Bulik CM, Berkman ND, Brownley KA, Sedway JA, Lohr $\mathrm{KN}$. Anorexia nervosa treatment: A systematic review of randomized controlled trials. Int J Eat Disord 2007; 40: 310-20.

28. Golden NH, Jacobson MS, Sterling WM, Hertz S. Treatment goal weight in adolescents with anorexia nervosa: Use of BMI Percentiles. Int J Eat Disord 2008; 41: 301-6.

29. Castro J, Gila A, Puig J, Rodríguez S, Toro J. Predictors of rehospitalization after total weight recovery in adolescents with anorexia nervosa. Int J Eat Disord 2004; 36: 22-30.

30. Lund BC, Hernández ER, Yates WR, Mitchell JR, McKee PA, Johnsonn CL. Rate of Inpatient Weight Restoration Predicts Outcome in Anorexia Nervosa. (Int J Eat Disord 2009; 42: 301-30.

31. Castro-Fornieles J, Casula V, Saura B, Martínez E, Lazaro L, Vila M, et al. Predictors of weight maintenance after hospital discharge in adolescent anorexia nervosa. Int J Eat Disord 2007; 40: 129-35.

32. Meguerditchian C, Samuelian-Massat C, Valéro R, Begu-Le Corroller A, Fromont I, Mancini J, et al. The impact of weight normalization on quality of recovery in anorexia nervosa. J Am Coll Nutr 2009; 28: 397-404.

33. National Institute for Health and Clinical Excellence. Nutrition support for adults. Oral nutrition support, enteral tube feeding and parenteral nutrition. London: National Institute for Clinical Excellence; 2006.

34. Silber TJ. A change of paradigm in the treatment of anorexia nervosa? Not so soon. J Adolesc Health 2008; 42: 109-10.

35. Mehler PS, Krantz M. Anorexia nervosa medical issues. J Womens Health 2003; 12: 331-40.

36. American Dietetic Association. Position of the American Dietetic Association: Nutrition intervention in the treatment of anorexia nervosa, bulimia nervosa, and other eating disorders. J Am Diet Assoc 2006; 106: 2073 82.

37. Herzog T, Zeeck A, Hartmann A, Nickel T. Lower targets for weekly weight gain lead to better results in inpatient treatment of anorexia nervosa: a pilot study. Eur Eat Disord Rev 2004; 12: 164-8.

38. Imbierowicz K, Braks K, Jacoby GE, Geiser F, Conrad R, Schilling G, et al. High-caloric supplements in anorexia treatment. Int J Eat Disord 2002; 32: 135-45.

39. Barbarich NC, McConaha CW, Halmi KA, Gendall K, Sunday SR, Gaskill J, et al. Use of nutritional supplements to increase the efficacy of fluoxetine in the treatment of anorexia nervosa. Int J Eat Disord 2004; 35: 10-15.

40. Birmingham L. Managing eating disorders: the refeeding syndrome. Can J Diagn 2008; 85-89.

41. Mehanna HM, Moledina J, Travis J. Refeeding syndrome: what it is, and how to prevent and treat it. BMJ 2008; 336: 1495-8

42. Crook MA, Hally V, Pantelli JV. The importance of the refeeding syndrome. Nutrition 2001; 17: 632-7.

43. Fisher M, Simpser E, Schneider M. Hypophosphatemia secondary to oral refeeding in anorexia nervosa. Int J Eat Disord 2000; 28: 181-7.

44. Tan JO A, Hope T, Stewart A, Fitzpatrick R. Control and compulsory treatment in anorexia nervosa: The views of patients and parents. Int J Law Psychiatry 2003; 26: 627-45.

45. Robb AS, Silber TJ, Orrell-Valente JK, Ellis N, ValadezMeltzer A, Dadson MJ. Supplemental nocturnal nasogastric refeeding for better short-term outcome in hospitalised adolescent girls with anorexia nervosa. Am J Psychiatry 2002; 159: 1347-53.

46. De Caprio C, Pasanisni F, Contaldo F. Gastrointestinal complications in patients with eating disorders. Eat Weight Disord 2000; 5: 228-30.

47. Practice guideline for the treatment of patients with 
eating disorders. American Psychiatric Association Work Group on Eating Disorders; 2006. doi:10.1176/ appi. books. 9780890423363.138660 .

48. Gentile MG, Manna GM, Ciceri R, Rodeschini E. Efficacy of inpatient treatment in severely malnourished anorexia nervosa patients. Eat Weight Disord 2008; 13:191-7.

49. Nogal P, Pniewska-Siark B, Lewiński A. Analysis of treatment efficacy in girls with anorexia nervosa (III). Neuro Endocrinol Lett 2009; 30: 32-8.

50. Couturier J, Mahmood A. Meal support therapy reduces the use of nasogastric feeding for adolescents hospitalized with anorexia nervosa. Eat Disord 2009; 17: 327-32.

51. American Psychiatric Association. Treatment of patients with eating disorders, third edition. Am J Psychiatry 2006; 163 (7 Suppl): 4-54.

52. Thomas B. Manual of dietetic practice, 4th ed. Oxford: Blackwell Publishers; 2007.

53. Jáuregui Lobera I, Bolaños Ríos P. Spanish version of the Irrational Food Beliefs Scale. Nutr Hosp 2010; 25: 852-9.

54. Setnick J. Micronutrient deficiencies and supplementation in anorexia and bulimia nervosa: A review of literature. Nutr Clin Pract 2010; 25: 137-42.

55. Robinson Y, Cristancho E, Böning D. Intravascular hemolysis and mean red blood cell age in athletes. Med Sci Sports Exerc 2006; 38: 480-3.

56. Birmingham CL, Gritzner S. How does zinc supplementation Benefit anorexia nervosa? Eat Weight Disord 2006; 11: e109-e111.

57. Birmingham CL, Goldner EM, Bakan R. Controlled trial of zinc supplementation in anorexia nervosa. Int J Eat Disord 1994; 15: 251-5.

58. Winston AP, Jamieson CP, Madira W, Gatward NM, Palmer RL. Prevalence of thiamin deficiency in anorexia nervosa. Int J Eat Dis 2000; 28: 451-4.

59. Folic acid recommendations page. Centers for Disease Control and Prevention Web site. http://www.cdc.gov/ ncbddd/folicacid/index. html. Accessed August 19, 2010.

60. Haagensen AL, Feldman HA, Ringelheim J, Gordon CM. Low prevalence of vitamin D deficiency among adolescents with anorexia nervosa. Osteoporos Int 2008; 19: 289-94.

61. Peters TE, Parvin M, Petersen C, Faircloth VC, Levine RL. A case report of Wernicke's encephalopathy in a pediatric patient with anorexia nervosa-restricting type. J Adolesc Health 2007; 40: 376-83.

62. Rock CL, Vasantharajan S. Vitamin status of eating disorder patients: relationship to clinical indices and effect of treatment. Int J Eat Disord 1995; 18: 257-62.

63. Capo-chichi CD, Guéant JL, Lefebvre E, Bennani N, Lorentz E, Vidailhet C, et al. Riboflavin and riboflavin- derived cofactors in adolescent girls with anorexia nervosa. Am J Clin Nutr 1999; 69: 672-8.

64. Vignini A, D’Angelo M, Nanetti L, Camilloni MA, Cester AM, Faloia E. Anorexia Nervosa: A role for L-Arginine supplementation in cardiovascular risk factors? Int J Eat Disord. 2010; 43: 464-71.

65. Dellava JE, Von Holle A, Torgersen L, ReichbornKjennerud T, Haugen M, Meltzer HM et al. Dietary supplement use immediately before and during pregnancy in Norwegian women with eating disorders. Int J Eat Disord 2011; 44: 325-32.

66. Nova E, Toro O, Varela P, López-Vidriero I, Morandé G, Marcos A. Effects of a nutritional intervention with yogurt on lymphocyte subsets and cytokine production capacity in anorexia nervosa patients. Eur J Nutr 2006; 45: 225-33.

67. Bozzetti F. Quality of life and enteral nutrition. Curr Opin Clin Nutr Metab Care 2008; 11: 661-5.

68. Malfi G, Agnello E, Da Pont MC, Palmo A, Zullo G, Monero A, et al. Chronic anorexia nervosa: enteral nutrition via percutaneous endoscopic gastrostomy. Minerva Gastroenterol Dietol 2006; 52: 431-5.

69. Neiderman M, Farley A, Richardson J, Lask B. Nasogastric feeding in children and adolescents with eating disorders: toward good practice. Int J Eat Disorder 2001; 29: 441-8.

70. Neiderman M, Zarody M, Tattersall M, Lask B. Enteric feeding in severe adolescent anorexia nervosa: a report of four cases. Int J Eat Disorder 2000; 28: 470-5.

71. Paccagnella A, Mauri A, Baruffi C, Berto R, Zago R, Marcon $\mathrm{ML}$, et al. Application criteria of enteral nutrition in patients with anorexia nervosa: correlation between clinical and psychological data in a "lifesaving" treatment. J Parenter Enteral Nutr 2006; 30: 231-9.

72. Robb AS, Silber TJ, Orrell-Valente JK, Valadez-Meltzer A, Ellis N, Dadson MJ, et al. Supplemental nocturnal nasogastric tube refeeding for better short-term outcome in hospitalizad adolescent girls with anorexia nervosa. Am J Psychiatry 2002; 159: 1347-53.

73. Silber TJ, Robb AS, Orrell-Valente JK, Ellis N, ValadezMeltzer A, Dadson MJ. Nocturnal nasogastric refeeding for hospitalizad adolescent boys with anorexia nervosa. J Dev Behav Pediatr 2004; 25: 415-8.

74. Zuercher JN, Cumella EJ, Woods BK, Eberly M, Carr JK. Efficacy of voluntary nasogastric tube feeding in female patients with anorexia nervosa. J Parent Enter Nutr 2003; 27: 268-76.

75. Rigaud D, Brondel L, Poupard AT, Talonneau I, Brun JM. A randomized trial on the efficacy of a 2-month tube feeding regimen in anorexia nervosa: A 1-year follow-up study. Clinical Nutrition 2007; 26: 421-9. 
76. Zuercher JN, Cumella EJ, Woods BK, Eberly M, Carr JK. Efficacy of voluntary nasogastric tube feeding in female inpatients with anorexia nervosa. JPEN J Parenter Enteral Nutr 2003; 27: 268-76.

77. Treasure J. A guide to the medical risk assessment for eating disorders. London: Maudsley Publications; 2004. data in a "lifesaving" treatment. JPEN J Parenter Enteral Nutr 2006; 30: 231-9.

78. Mehler PS, Weiner KL. Use of total parenteral nutrition in the refeeding of selected patients with severe anorexia nervosa. Int J Eat Disord 2007; 40: 285-7.

79. Diamanti A, Basso MS, Castro M, Bianco G, Ciacco E, Calce A, et al. Clinical efficacy and safety of parenteral nutrition in adolescent girls with anorexia nervosa. J Adolesc Health 2008; 42: 111-8.

80. Melchior JC, Corcos M. Parenteral Nutrition and Anorexia Nervosa: Is it useful, is it ethical? J Adolesc Health 2009; 44: 410-2.

81. Zaloga GP. Parenteral nutrition in adult inpatients with functioning gastrointestinal tracts: Assessment of outcomes. Lancet 2006; 367: 1101-11.

82. Kochevar M, Guenter P, Holcombe B, Malone A, Mirtallo J. A.S.P.E.N. Statement on Parenteral Nutrition Standardization. JPEN J Parenter Enteral Nutr 2007; 31: 441-8.

83. Miller SJ. Death resulting from overzealous total parenteral nutrition: the refeeding syndrome revisited. Nutr Clin Pract 2008; 23:166-71.

84. Marinella MA. Refeeding syndrome and hypophosphatemia. J Intensive Care Med 2005; 20: 155-9.

85. Solomon SM, Kirbi DF. The refeeding syndrome. A review. J Parenter Enteral Nutr 1990; 14: 90-7.

86. Tey HL, Lim SC, Snodgrass AM. Refeeding oedema in anorexia nervosa. Singapore Med J 2005; 46: 308-10.

87. O'Connor G, Goldin J. The refeeding syndrome and glucose load. Int J Eat Disord 2011; 44: 182-5.

88. Katzman DK. Medical complications in adolescents with anorexia nervosa: A review of the literature. Int J eating Disord 2005; 37: S52-S59.

89. Hearing SD. Refeeding syndrome. BMJ 2004; 328: 908-9.

90. Guidelines for the prevention and treatment of adult patients at risk of developing refeeding syndrome. Drug Therapy Guideline No. 46.00. In: Refeeding Syndrome Guideline. NSH; October 10, 2007.

91. De Cock A, Mana F, Velkeniers B, Urbain D. Hypophosphatemia and refeeding: a corrective or a preventive attitude? Acta Clin Belg 2006; 61: 134-7.

92. Thiels C. Forced treatment of patients with anorexia. Curr Opin Psychiatry 2008; 21: 495-8.

93. Treasure J, Schmidt U, Furth E. The Essentials handbook of eating disorders. Chichester, West Sussex: John Wiley \& Sons; 2005.

94. Serfaty M, McClusky S. Compulsory treatment of anorexia nervosa and the moribund patient. Eur Eat Disord Rev 1998; 6: 22-37.

95. Vialettes B, Samuelian-Massat C, Valero R, Béliard S. The refusal of treatment in anorexia nervosa, an ethical conflict with three characters: "The girl, the family and the medical profession”. Diabetes Metab 2006; 32: $306-$ 11.

96. UK Parliament. Mental Health Act 2007 (c 12). London: The Stationery Office; 2007.

97. Carney T, Wakefield A, Tait D, Touyz S. Reflections on coercion in the treatment of severe anorexia nervosa. Isr J Psychiatry Relat Sci 2006; 43; 159-65.

98. Carney T, Tait D, Richardson A, Touyz S. Why (and when) clinicians compel treatment of anorexia nervosa patients. Eur Eat Disord Rev 2008; 16: 199-206.

99. Gómez Candela C, De Cos Blanco AI, Armero Fuster M, Calvo Viñuela I, Díaz Gómez J, Rico MA, et al. Trastornos de la Alimentación: Programa de Educación Nutricional. Unidad de Nutrición Clínica y dietética. Madrid: Hospital Universitario La Paz; 2004.

100. Dwyer J T. Role of the nutritionist in eating disorders. New Dir Ment Health Serv 1986; 31: 21-8.

101. Breen HB, Espelage DL. Nutrition expertise in eating disorders. Eat Weight Disord 2004; 9: 120-5.

102. Loria Kohen V, Gómez Candela C, Lourenço Nogueira T, Pérez Torres A, Castillo Rabaneda R, Villarino Marin M, et al. L. Evaluation of the utility of a nutrition education program with eating disorders. Nutr Hosp 2009; 24: 558-67. 